\title{
Noch einmal „Ellä toledoth“.
}

Von Prof. D. Karl Budde in Marburg.

In seiner Heidelberger Dissertation „Die Priesterschrift in der Genesis" (Halle [Saale] 1915) stellt lic. Walther Eichrodt S. 23 Fubnote I fest, daß mein Aufsatz Ellä toledoth (hier 19I4 S. 24I ff.) erst nach Fertigstellung seines Manuskripts erschienen sei und daher nur in einem Nachtrag (S. 52-54) habe berücksichtigt werden können. Mir will es scheinen, als wenn es richtiger gewesen wäre, die Tatsache des Zuspätkommens meiner Arbeit einfach hinzunehmen, als ihr eine so flüchtige und irreführende Berücksichtigung zuteil werden zu lassen. Denn nur Flüchtigkeit kann es erklären und entschuldigen, wenn E. S. 53 behauptet, ich ,glaubte das Schema für alle [Toledoth-] Überschriften auf Grund von Gen 25 ig feststellen zu können".x Er führt dafür, abweichend von seinem sonstigen Verfahren, keine Beweisstelle an. Er kann nur S. 247 meinen, wo ich in der Tat 2519 für die Herstellung von II to verwende. Dort aber sage ich ganz unmißverständlich: „Es sollte nach der allgemeinen Regel und nach dem Wortlaut von 25 ig insbesondere heißen" usw. Ich unterscheide also zwischen 1. der allgemeinen Regel und 2. dem Muster, das für diesen besonderen Fall in 25 ig vorliegt, darum aber entfernt nicht für alle Überschriften. Die allgemeine Regel aber steht auf derselben Seite oben, da@ nämlich hinter der Überschrift eine Wiederholung des in Betracht kommenden Ausschnitts aus dem vorherigen Hauptstück das neue einleite. Von dem vorherigen Hauptstück also hängt es ab, was hinter der Überschrift diesem Zwecke dienstbar gemacht wird, nicht von 25 19; danach ist es klar, daß nach meiner Anschauung Inhalt und Wortlaut dieses "passus concernens" bei den verschiedenen Haupt-

I Nach den weiteren Ausführangen will er damit sagen, ich glanbte das Schema für alle in $25 \times 9$ gefunden $z \mathrm{a}$ haben. So "wiederholt er selbst weiter unten: „25 29 ist BuDdes Musterbeispiel.“ Auch S. 54 stellt er abschliefend wieder dasselbe fest.

Zeitschr. E d. alttest. Wiss. Jahrg. 36. 1926. 
stuicken ein himmelweit verschiedener sein kann und nur da, wo die Verhältnisse ganz gleich liegen, eine Stelle für die andere als Muster herangezogen werden darf. Nur auf die Wiederholung als solche geht auch der weitere Satz auf derselben Seite, den E. S. 53 Fußnote I wörtlich anzieht: „Ob dieses Gesetz ursprünglich auch nur eine einzige Ausnahme zugelassen hat, mag man billig fragen." Er aber entnimmt daraus im Text, zu dem er die Stelle als Beweis gibt, dab ich mich "auf so absolute Regelmäßigkeit in den Úberschriften P's versteife“. Wie ist das nur möglich, da ich eben vorher als Beispiele 10 I "Die Söhne Noahs waren'Sem, Ham und Japhet", 2512 "Sohn Abrahams, welchen Hagar die Ägypterin, die Magd Saras, dem Abraham geboren hatte“, und 25 19 „Abraham zeugte den Isaak“, also alle verschieden voneinander, im vollen Wortlaut aufführe? Aber ohne Gnade legt mich nun E. auf 25 ig fest $^{x}$ und verzeichnet bei allen zehn Überschriften jede Abweichung diesem Schema gegenüber als Beweis gegen meine Meinung; kaum daß er für 5 I eine Ausnahme, für 2512 die Berechtigung einer kleinen Abweichung zugibt. Mit solchem Verfahren den Gegner zu widerlegen, ist freilich kein Kunststück; in Wirklichkeit aber widerlegt man so nur sich selbst in seinem Irrtum.

Damit aber diese notgedrungene Verwahrung nicht ohne sachlichen Gewinn bleibe, will ich nicht unterlassen, den Einwänden EICHrodTs, soweit sie die Möglichkeit dazu bieten, nachzugehn. Da er in der Nachfolge von EERDMans und SMEND die Hauptstücküberschriften für $\mathrm{Pg}^{2}$ preisgibt, möchte sonst dennoch der Eindruck entstehn, als wenn durch seine Schrift diese Anschauung an Wahrscheinlichkeit gewonnen hätte. Zugleich aber wird auch zutage kommen, was etwa auf seiner Seite anzuerkennen, auf meiner zu verbessern ist.

$\mathrm{Ob}$ es schwerer ist, für gleichförmige Überschriften oder im Lauf der Erzählung dasselbe Wort in wechselnder Bedeutung zu gebrauchen (S. 52 Fußnote), wird sich nicht grundsätzlich entscheiden lassen; für den Gebrauch von תִ ה 24 a darf ich zur Ergänzung auf meinen

× Er fährt S. 53 oben wörtlich in der Wiedergabe meiner Meinung fort: „Nach den Worten ואלה חist zolgt züchst die Angabe des Vaters des Betreffenden, und zwar in der ebenfalls stereotypen Wendung "פ" הוליד אתדפ, dann ev. eine kurze Notiz über die Hauptperson, etwa ihr Alter 0 . a.,, dann die Aufzählung der Nachkommen." Nicht ein Wort von alledem steht in meinem Aufsatze. Das Zitieren und Referieren wird E. doch wohl noch lernen mūssen.

2 Ich berichtige kier bei Gelegenheit das Flüchtigkeitsversehen, durch das ich auf S. 90 die Bezeichnung. Pg Wellhausen zueigne. Sie ist bekanntlich von Holzinger eingeführt. 
Aufsatz "Wortlaut und Werden" usw. (hier 1915) S. 66 verweisen. Für 5 I 2 hatte ich selbst a. a. O. S. 247 Verbesserungen freigegeben; in dem neuen Aufsatz S. 8o Anm. I habe ich für die wesentlichste einen Vorschlag gemacht. Was 69 angeht, so kann man E. soweit recht geben, daß die Charakteristik Noahs etwas Auffallendes hat. Nicht etwa, weil sie das mir nur aufgedrungene enge Schema störte, sondern weil sie Neues an der Stelle bringt, wo wir schon Berichtetes, eben den passus concernens, erwarten sollten, und es obendrein in einer Fassung bringt, als wenn in der Tat schon Berichtetes angezogen würde. ${ }^{x}$ Man hat ein Recht daraus zu schließen, daß bei 532 dergleichen wirklich schon gesagt war, dort aber verloren gegangen oder von der Redaktion gestrichen worden ist. Doppelt rechtfertigt sich diese Annahme, wenn wir bedenken, daß wesentlich dasselbe, mit der gleichen Wendung, die in 69 den Schluß bildet, bei Henoch (5 [22] 24) geflissentlich hervorgehoben ist. In meiner Biblischen Urgeschichte habe ich nachgewiesen (S: $93 \mathrm{ff}$.), daf für $\mathrm{P}$ die erste Hälfte der Geschlechter der Sethitentafel als gut, die zweite mit Ausnahme des siebenten Geschlechtshaupts, Henoch, und des zehnten, Noah, als böse und damit dem Verderben durch die Sintflut verfallen gilt; das beweisen die ursprünglichen Zahlen des Samaritanus und die Namen in ihrer eigenartigen Ausgestaltung. Eben deshalb wird die Ausnahme auch bei Noah hervorgehoben gewesen sein, vielleicht diesmal, anders als bei Henoch, vor der Erwähnung der Söhne, die er ja erst mit 500 Jahren zeugte. ${ }^{2}$ Wodurch dieser Absatz verloren gegangen ist, können wir nicht mehr feststellen. Vielleicht genügte, wenn man von bloßem Textschaden absehen will, das Dazwischentreten der so weit abseits führenden Engelehen $6 \mathrm{I}-4$; vielleicht auch hat man auf Grund einer abweichenden Überzeugung die Betonung der Verderbtheit der letzten Sethitengeschlechter an dieser entscheidenden Stelle mit vollem Bedacht getilgt. Das wäre doppelt begreiflich, wenn. etwa in 532 neben der Frömmigkeit Noahs auch die Verderbtheit seiner Umgebung deutlicher noch als in dem vạ von 69 zu lesen stand. 3 Aber E. hat hier noch eine andre Einwendung zu machen. Warum fehlt in dem passus

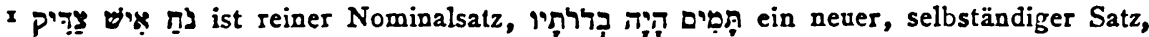
etwa "als untadelig hatte er sich erwiesen unter seinen Zeitgenossen."

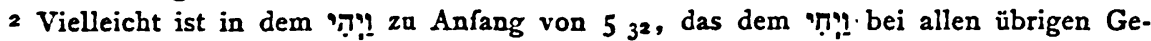
schlechtern als einzige Ausnahme gegenübersteht, noch der Ansatz zu dieser Aussage erbalten geblieben.

3 Auch die Verwirrang des Wortlauts von 5 22-24 könnte mit dergleichen 'zu schaffen haben. 
conccrnens die Nennung des Vaters Noahs? Oben schon habe ich es abgelehnt, mich dafür überall auf 25 ig festnageln zu lassen. Aber einen Grund dafür haben wir soeben kennen gelernt. Es hatte nichts Verlockendes, den zum Untergang in der Sintflut verurteilten Vater Noahs von neuem aufzuführen. Dazu kommt doch wohl der andre Grund, dab in diesem Falle das vorhergehende Hauptstuick bereits über den Helden des neuen hinausgeschritten war zu den drei Söhnen, die ohnedies für das Folgende allein von Bedeutung, waren. Es wird gut sein, das auch ferner im Auge zu behalten.

Auch dab ich in IO I den passus concernens durch Einschub -

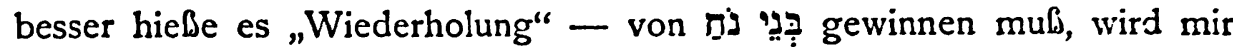
auf das Debetkonto geschrieben (vgl. auch S. 54). E. drückt sich hier so vorsichtig aus, daß man sich des Eindrucks kaum erwehren kann, $\mathrm{dab}$ er die Herstellung selbst billigt; trifft das $z \mathrm{u}$, wie kann sie dann gegen mich zeugen? Wenn E. mich wieder damit zu treffen meint,

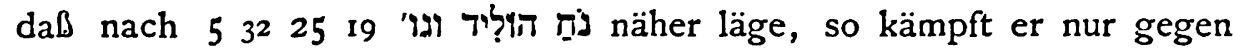
seine Windmühle. Aber außerdem wei@ er doch wohl auch, auf welche Weise die Quellenscheidung in der Völkertafel endlich geglückt ist, nämlich durch Wellhausens Entdeckung, daß $P$ hier durchgängig nicht die

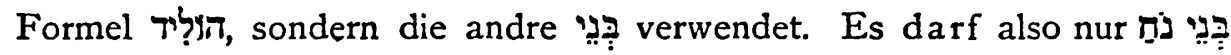
ergänzt werden, ganz abgesehen davon, daß damit der Ausfall sich spielend leicht erklärt.

Bei II to vergißt E. neben der Feststellung, daß hier „die geבן נח נח הוליד את-שם ויהי wünschte Wiederaufnahme durch Einschub von hergestellt werden müsse", daß ich hier die Möglichkeit einer Ausnahme, des Fehlens also des passus concernens, nachdrücklich freigegeben habe, zweimal sogar, auf S. 247 und 248 . War er mir nicht schuldig, das zu erwähnen? Einen Grund für. dieses Zugeständnis gebe ich dort nicht an; es ist aber keineswegs willkürlich gemacht, schädigt auch nicht die Geltung der Regel. Wie ich daneben freigab, daß ein Redaktor den für die Ergänzung vorgeschlagenen Absatz „so dicht hinter IO I gestrichen haben möchte", so schien es mir auch denkbar, daßs sogar $\mathrm{Pg}$ selbst angesichts von 5326 10 10 I und unmittelbar hinter seiner Semitentafel ro 22f. 32 f. die Wiederholung diesmal für vollkommen überflüssig gehalten und unmittelbar mit שם בן־מאת שנה וגו' eingesetzt hätte. Und dennoch muß ich das jetzt aus einem anderen Grunde, den ich damals übersah, für ganz unwahrscheinlich erklären. Die Semitentafel II 10-26 zählt von Sem bis Terach nur neun Glieder, während nach dem Beispiel von Kap. 5 deren zehn umsomehr zu erwarten sind, 
als J Arpakhschad und Schelach nicht kennt (vgl. IO 21 25), diese also wahrscheinlich hinzugefügt wurden, um die runde $Z$ ahl zu erreichen. Der Kenan, den LXX zwischen jenen beiden einschiebt, muß als apokryph geiten, Abraham als zehnten hinzuzunehmen ${ }^{3}$, ist schon wegen der Dreiteilung hinter Terach schwerlich zulässig. So kam ich schon in meiner „Biblischen Urgeschichte“ S. 4I I ff. zu dem Schluß, daß vielmehr Noah hier ebenso wie in der Sethitentafel mitgezählt sei. Dafür bot ich dort die sachliche Rechtfertigung. Der Form nach aber rechtfertigt sich diese Annahme erst dann, wenn man, was ich jetzt unter einem ganz andren Gesichtspunkt vorzuschlagen Anlaß fand, die Abstammung Sems von Noah hinter der Überschrift herstellt.

Weiter versucht EICHRODT SMENDs Befremden, daß hinter den Toledoth der Söhne Noahs noch die Sems folgen, das ich nicht zu verstehn bekannt hatte, seinerseits $\mathrm{zu}$ rechtfertigen. Eine ähnliche Wiederaufnahme finde sich sonst nirgends, während man sie bei den Söhnen Jakobs und Josef doch erwarten könnte. Er biegt dann aber SMENDs Anstoß dahin um, dab vielmehr nach den sonstigen Gepflogenheiten des Toledothschreibers statt der Toledoth. der Söhne Noahs solche von Japheth und Ham einzeln, vor denen Sems - ich setze dies letzte, gewi@ in seinem Sinne, hinzu - zu erwarten wären.

Ich glaube, $\mathrm{Pg}$ hat genau gewußt, was er tat, als er dieser Erwartung, die sich auf Ismael vor Isaak, Edom vor Jakob berufen kann, nicht entsprach. Hätte er es getan, so hätte er den ganzen Inhalt von $1022 \mathrm{f}$. mit in die Toledoth Sems unterstopfen müssen, und davon konnte ja bei dem festen -Schema der Stammtafel der auserwählten Linie unter Anschluß der Weltzeitrechnung, der genauen Fortsetzung. des zweiten Hauptstücks Kap. 5, gar keine Rede sein.' 'Elam, Aššur, Lud, Aram, 'Uș, Chul, Geter und Maš gehören ihm durchaus in die Gemeinschaft mit sämtlichen Nachkommen Jepheths und Chams und haben in der Tafel der auserwählten Linie gar nichts zu suchen; es war daher einfach das Gegebene, die „Völkertafel" zu schaffen, in ihr die Welt mit einem Male abzutun und über sie zur Tagesordnung der Auserwählten überzugehn. Hier hat SMEND viel mehr Recht als EICHRODT. Sollte die Wiederholung Sem und Sem (IO 21 und II 9) vermieden werden, so mubte nicht an Kap. 10 geändert werden,. sondern

I So z. B. A. Kueven, De stamboom van den masoretischen text des O. Testaments, S. 48 .

2 Das weif E. S. 46 im Grande so gut, dal man sich wundern muf, wie er daran irre werden kann. 
an Kap. 11, indem Arpakhschad statt seines Vaters an die Spitze gestellt wurde. Aber die Zehnzahl ging damit verloren, wenigstens bei dem jetzigen Bestand an Namen, und ob weitere zur Verfügung standen, wissen wir nicht. $O b$ wir aber $P$ überhaupt zumuten dürfen, dab die Wiederholung ihm Bedenken gemacht hätte, scheint mir mehr als zweifelhaft. Wie sich E. denkt, was er von den Söhnen Jakobs und Joseph sagt, bekenne ich wieder nicht zu verstehn.

Hat sich also für II 9 die Änderung nach dem Muster von 25 I9 durchaus bewährt, so bin ich heute nicht mehr so sicher, ob dasselbe Verfahren auch bei 1127 Stich hält. Es läbt sich doch nicht verkennen, daß hier der Vergleich mit 69 näher liegt; denn an beiden Stellen spaltet sich der Stammbaum des vorhergehenden Hauptstücks dicht vorher in drei Äste. Wenn nun 69 die Toledoth Noahs, des Vaters der drei, eröffnet, so entspricht es dem völlig, wenn in 1127 Terach, nicht Abraham, einer von seinen drei Söhnen, an der Spitze steht. Freilich war bei Noah ein andres Verfahren ganz ausgeschlossen, während in Kap. $12 \mathrm{ff}$. Abraham ebenso die Hauptfigur ist wie Noah in Kap. 6-9, und ich bin, ganz anders als E. denkt, der letzte, der $\mathrm{Pg}$ auf sein engstes Schema unerbittlich festlegen möchte. Aber freigeben muß ich doch unter diesen Umständen neben der Änderung in

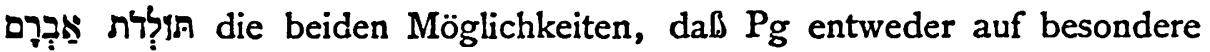
"Toledoth Abrahams" arglos verzichtet hat, oder daß diese Überschrift hinter Kap. II irgendwo ausgefallen ist, so daß sich II Hauptstücke statt ro ergäben. Von diesen Möglichkeiten würde mir die erste wahrscheinlicher dünken, nicht wegen der runden Zahl, sondern weil $R$ im Anfang der Genesis mit $P$ überhaupt, vor allem aber mit diesen Überschriften, so schonend umgegangen ist, da $\$$ man wenigstens ihm die Beseitigung schwerlich zutrauen dürfte.

Von meiner Herstellung von 372 erklärt sich E. nicht überzeugt, ohne Gründe dafür anzugeben; ich brauche daher die meinigen nicht zu wiederholen. Ebenso macht er es gegenüber meiner genauen Anführung der Stellen, in denen ich ausreichende Spuren der Beteiligung P's an der Geschichte der Geburt der Jakobsöhne finde (S. 250). Auch aus seiner Verweisung "vergleiche oben zur Stelle" habe ich leider nichts gelernt, da ich wieder nichts anderes finden konnte als die lakonische Feststellung S. 19, daß "hier" - augenscheinlich in Kap. 2930 - „bei Pg eine Lücke klafft.“ Nur eine Bemerkung bietet etwas von einer Handhabe. E. meint, es sei ganz unklar, was der ganze Vers 372 in $\mathrm{Pg}$ bedeuten solle, da Pg den Streit der Brüder, zu dem $372 \mathrm{~b}$ 
die Einleitung bilde, wahrscheinlich überhaupt nicht erwähnt habe. Darüber kann man jedenfalls verschiedener Meinung sein. BACON, den man bei diesen Dingen nie unbefragt lassen sollte, ergänzt den $\mathrm{Zu}$ sammenhang von 372 und 4I 46 für P: „Als Joseph siebzehn Jahre alt war [ging er mit seinen Brüdern hinaus aufs Feld. Und seine Brüder verkauften ihn nach Ägypten, und er war dort zwölf Jahre in Banden. Aber Pharaoh, der König von Ägypten, hörte von der Weisheit Josephs und machte ihn zum Regenten, über das Land]. Und Joseph war dreißig Jahre alt usw." Indessen ist es sehr möglich, dab $P$ hier wie bei Ismael und Edom alles schiedlich-friedlich hat zugehn lassen. An der Sache würde das nichts ändern; denn nach Ägypten ist Joseph auch nach $\mathrm{P}$ gekommen, und die I7 Jahre gäben dann nur an, wann eine Räuberhorde ihn dorthin verschleppt und verkauft hätte. Dann wäre etwa nur v. 2 a $\alpha$ (bis v. 2 a $\beta$ hatte ich S. 25 I Anm. 2 bereits freigegeben. Im übrigen dürfte BACONs Herstellung der.Hauptsache nach das Richtige treffen.

Dankenswert ist, daß E. wenigstens die Zeitrechnung für Pg festhält; aber gerade, sie wird sich ohne die Hauptstücküberschriften schwer verteidigen lassen. Vor allem aber bleibt es nach wie vor dabei, dab deren Einschiebung durch die Hand eines Redaktors, der das Ganze übersah, obendrein vielfach an Stellen, die für das Mosaik der Quellen so überaus ungeschickt gewählt erscheinen, ganz undenkbar ist. Tapfer genug hat E. sich gewehrt gegen die bohrende Zähigkeit, mit der EERDMANS seine Divide et impera-Politik durchführt; aber doch hat er wie SMEND sich dadurch weiter drängen lassen, als durch die Sache gerechtfertigt wird. Keine der Stellen, die er P aberkennt oder doch für zweifelhaft erklärt, hätte er, soweit ich zu sehen vermag, preiszugeben brauchen.

Nachschrift yom I 3. Januar I9r6. Seit Einsendung der Druckvorlage erschien als Nr. $3^{*}$ der Beihefte zu dieser Zeitschrift EICHRODTs Buch „Die Quellen der Genesis von neuem untersucht", Gießen I9I6. Da der Verf. darin seine, zu Eingang angeführte Dissertation unverändert als ersten Teil abdruckt, auch nicht weiterhin auf die oben behandelten Fragen zurückkommt, so wird meine Entgegnung auch heute noch ihre Dienste tun. Die Seitenzahlen der Anführungen stimmen auch für. das .vollständige Buch. 